\title{
Phytochemical Constituents and the Evaluation Biological Effect of Cinnamomum yabunikkei H.Ohba Leaf
}

\author{
Seung-Yub Song ${ }^{1}$, Seung-Hui Song ${ }^{1}$, Min-Suk Bae ${ }^{2}$ (I) and Seung-Sik Cho ${ }^{1, *(1)}$ \\ 1 Department of Pharmacy, College of Pharmacy, Mokpo National University, Muan, Jeonnam 58554, Korea; \\ tgb1007@naver.com (S.-Y.S.); tmdgm17898@naver.com (S.-H.S.) \\ 2 Department of Environmental Engineering, Mokpo National University, Muan, Jeonnam 58554, Korea; \\ minsbae@hotmail.com \\ * Correspondence: sscho@mokpo.ac.kr; Tel.: +82-61-450-2687
}

Academic Editors: In-Soo Yoon and Hyun-Jong Cho

Received: 13 December 2018; Accepted: 24 December 2018; Published: 27 December 2018

\begin{abstract}
Cinnamomum yabunikkei H.Ohba leaf is known as a traditional medicinal material in Korea. However, no scientific identification of the components or efficacy of C.yabunikkei H.Ohba leaf has been reported. In the present study, we prepared various solvent extracts of C.yabunikkei H.Ohba leaf to understand its basic properties and evaluated the antioxidant, xanthine oxidase inhibitory, and elastase inhibitory activities of hexane, ethyl acetate, acetone, methanol, ethanol, and water extracts for the first time. The antioxidant properties were evaluated based on 1,1-diphenyl-2-picrylhydrazyl (DPPH) free radical scavenging activity, reducing power, and total phenolic contents. The hot water extract showed the highest DPPH radical scavenging activity and total phenolic contents, and the reducing power was the highest in the water extract. The hexane extract showed an excellent elastase inhibitory effect compared to control (phosphoramidone) and the highest xanthine oxidase inhibitory activity. These results present basic information for the possible uses of the hot water and hexane extracts from C. yabunikkei leaf for the treatment of diseases caused by oxidative imbalance. In the present study, individual extracts exhibited different effects. Therefore, it is hypothesized that the applicability of $C$. yabunikkei will depend on the extraction method and nature of the extract. The hot water and hexane extracts could be used as antioxidants, and as anti-gout and anti-wrinkle materials respectively. Several biologically active substances present in hexane extract of C. yabunikkei have been analyzed by GCMS and demonstrated to possess antioxidant and xanthine oxidase inhibitory activity. To the best of our knowledge, this is the first study that reports the chemical profiling and biological effects of various C. yabunikkei leaf extracts, suggesting their potential use in food therapy, cosmetics or alternative medicine.
\end{abstract}

Keywords: Cinnamomum yabunikkei leaf; antioxidant; xanthine oxidase; elastase

\section{Introduction}

Cinnamomum yabunikkei $\mathrm{H}$.Ohba is a species of camphor tree native to the southern coast and Jeju Island in South Korea and can be seen in southern China and Taiwan. Leaves have been used as a bath material or tea and berries as a source of oil. In addition, the bark has traditionally been used for the treatment of various diseases related to pain and blood circulation.

Due to the acceleration in global warming, the southern part of South Korea has experienced the cultivation of C.yabunikkei H.Ohba, hence studies dealing with the possibilities of using C.yabunikkei H.Ohba for various purposes are necessitated. 
Until now, the components of the leaves of C.yabunikkei have not been identified and biological activity studies have not been conducted. To the best of our knowledge, this is the first report to understand the composition and biological activity of the C.yabunikkei leaf.

In the present study, various extracts of the C.yabunikkei leaf using hexane, ethyl acetate, acetone, ethanol, methanol, and hot water were prepared to determine the optimal extraction solvent with respect to biological activity and phytochemical profiles. Gas chromatography-mass spectrometry (GC-MS) was used for the chemical profiling of hexane extracts. Subsequently, antioxidant, xanthine oxidase inhibitory, and elastase inhibitory activity of the leaf extracts were examined. The antioxidant activity was confirmed by measuring 1,1-diphenyl-2-picrylhydrazyl (DPPH) radical scavenging activity, reducing power, and reducing total phenolic contents.

\section{Results and Discussion}

\subsection{Antioxidant Activity and Total Phenolic Contents of C.yabunikkei Extracts}

The antioxidant potential of various extracts of the C.yabunikkei leaf was determined by measuring 2,2-diphenyl-1-picrylhydrazyl (DPPH) scavenging activity, reducing power assay and reducing total phenolics. The DPPH scavenging assay is a fast and easy method for evaluating the free radical scavenging capacity of given samples. The antioxidant effects of extracts are generally related to the phenolic contents, and phenolic-rich sources of phytochemicals with antioxidant activity have curative benefits against conditions such as inflammation, oxidative stress, and other metabolic diseases [1]. The antioxidative properties of the test plant extracts were closely correlated with the composition of active compounds such as phenolics.

Therefore, we compared the phenolic contents (mg/g as gallic acid) of various C.yabunikkei leaf extracts. The DPPH radicalscavenging activity is shown in Figure 1. The hot water, methanol, and ethanol extracts showed the highest DPPH radical scavenging activity $(81.4 \pm 0.8 \%, 80.6 \pm 2.1 \%$, $82.89 \pm 3.1 \%$ ) at the concentration of $0.5 \mathrm{mg} / \mathrm{mL}$. Ascorbic acid (Vit C, $20 \mu \mathrm{g} / \mathrm{mL}$ ) was used as positive control.

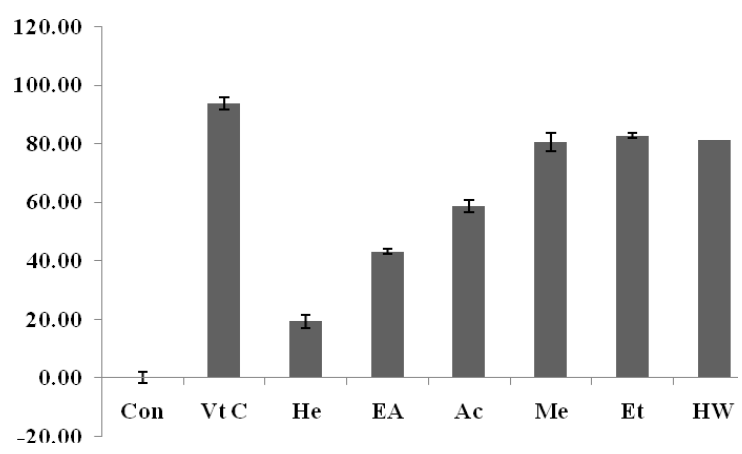

Figure 1. DPPH scavenging activity of solvent extracts of C.yabunikkei leaf (extract cont: $0.5 \mathrm{mg} / \mathrm{mL}$, Ascorbic acid: $20 \mu \mathrm{g} / \mathrm{mL}$ ). The asterisk represents a value significantly different from the other groups $(p<0.05)$. Values were the mean \pm standard deviation $(n=3) \mathrm{Vt} C$; ascorbic acid, He; hexane ex, EA; ethylacetate ez, Ac; acetone ex, Me; methanol ex, Et; ethanol ex, HW; hot water ex.

The reducing power assay is useful for evaluating the antioxidant activity. In the present study, the reductive capability of extract samples was tested by measuring the reduction of $\mathrm{Fe}^{3+}$. The hot water extract exhibited the highest activity compared to the other extracts (Table 1). The reductive activity expressed as vitamin C equivalents was $3.27 \pm 0.052 \mu \mathrm{g} / 100 \mu \mathrm{g}$ as the extract.

The total phenolic content was determined by Folin-Ciocalteu method [1], and the data was reported as gallic acid equivalents by referencing the standard curve $\left(r^{2}>0.999\right)$, as shown in Table 1. The phenolic content of the hot water extract was higher than that of the other extracts $(16.39 \pm 0.28 \mathrm{mg} / \mathrm{g}$ as gallic acid equivalents). Taken together, the results indicate that the DPPH 
radical scavenging activity, power reduction, and phenolic contents reduction were significantly higher in the hot water extract as compared to other extracts.

Table 1. Reducing power and total phenolic contents of C.yabunikkei leaf extracts.

\begin{tabular}{ccc}
\hline Extract & $\begin{array}{c}\text { Reducing Power (Ascorbic Acid eq. } \\
\boldsymbol{\mu g} / \mathbf{1 0 0} \boldsymbol{\mu g} \text { Extract) }\end{array}$ & Total Phenolic Content (Gallic Acid eq. $\mathbf{~ m g / g )}$ \\
\hline He & $0.25 \pm 0.002$ & $0.06 \pm 0.00$ \\
EA & $0.55 \pm 0.003$ & $0.98 \pm 0.00$ \\
Ace & $1.28 \pm 0.018$ & $6.18 \pm 0.19$ \\
Me & $2.73 \pm 0.068$ & $9.24 \pm 0.17$ \\
Et & $2.05 \pm 0.034$ & $13.89 \pm 0.45$ \\
HW & $3.27 \pm 0.052$ & $16.39 \pm 0.28$ \\
\hline
\end{tabular}

\subsection{Xanthine Oxidase Inhibitory Activity of C.yabunikkei Leaf Extracts}

The effect of various solvent extracts on the xanthine oxidase inhibitory activity of the C.yabunikkei leaf is shown in Figure 2. Allopurinol (ALP, positive control) at a concentration of $50 \mu \mathrm{g} / \mathrm{mL}$ significantly inhibited xanthine oxidase activity $(94.54 \%)$. The xanthine oxidase inhibitory activity of the hexane extract was significantly higher than other extracts at the concentration of $1 \mathrm{mg} / \mathrm{mL}$ (19.93\%). Previously, we reported four different botanical extracts as potential xanthine oxidase inhibitors [2]. Yoon et al. [3,4] reported that the optimized extracts of Corylopsis coreana and Camellia japonica inhibited xanthine oxidase activity by approximately $50 \%$ at a concentration of $2 \mathrm{mg} / \mathrm{mL}$. Additionally, Yoon et al. [2] demonstrated that Quercus acuta extract showed approximately 50\% xanthine oxidase inhibitory activity at a concentration of $1 \mathrm{mg} / \mathrm{mL}$. Cudrania tricuspidata extract inhibited xanthine oxidase by approximately 75\% at a concentration of $2 \mathrm{mg} / \mathrm{mL}$ [5]. Previously, we have screened four kinds of xanthine oxidase inhibiting sources in five hundred Korean plant extracts (data not shown). The activities of hexane extract were similar to the extracts of Corylopsis coreana and Camellia japonica, but about 2.5 times lower than Quercus acuta extract and about 1.8 times lower than Cudrania tricuspidata extract. The plant extracts with $\mathrm{XO}$ inhibitory activity at 1 and $2 \mathrm{mg} / \mathrm{mL}$ demonstrated consistent effects in the in vivo animal disease model. Thus, it is plausible that the hexane extract of C.yabunikkei leaves could be developed as a candidate anti-hyperuricemic agent.

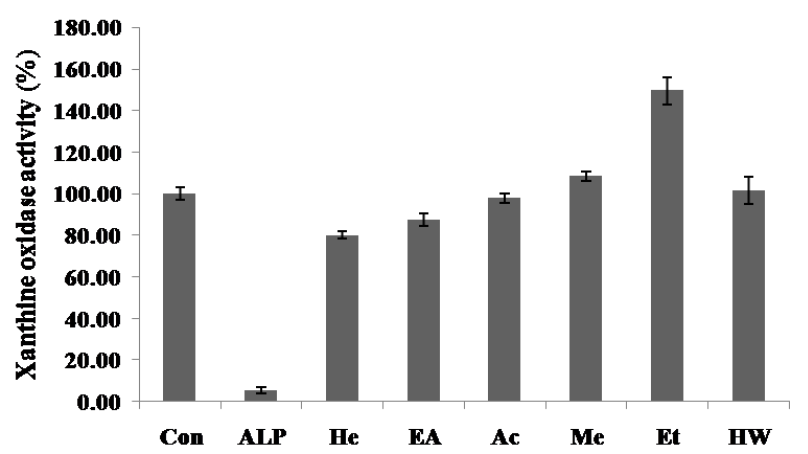

Figure 2. Xanthine oxidase inhibitory activities in various solvent extracts of C.yabunikkei leaves $(1 \mathrm{mg} / \mathrm{mL}$ ) and allopurinol (ALP, $50 \mu \mathrm{g} / \mathrm{mL}$ ). ALP; allopurinol, He; hexane, EA; ethylacetate, Ac; acetone, Me; methanol, Et; ethanol, HW; hot water extract. Values were the mean \pm standard deviation $(n=3)$.

\subsection{Elastase Inhibitory Activity of C.yabunikkei Leaf Extracts}

The effect of various solvent extracts on the elastase inhibitory activity of C.yabunikkei leaf is shown in Figure 3. Phosphoramidon (PPRM, positive control) at a concentration of $0.5 \mathrm{mg} / \mathrm{mL}$ significantly inhibited elastase activity $(56.12 \pm 1.53 \%)$. The elastase inhibitory activity of the hexane extract was 
significantly higher than other extracts and positive control at the concentration of $0.5 \mathrm{mg} / \mathrm{mL}(75.65$ $\pm 3.5 \%)$.

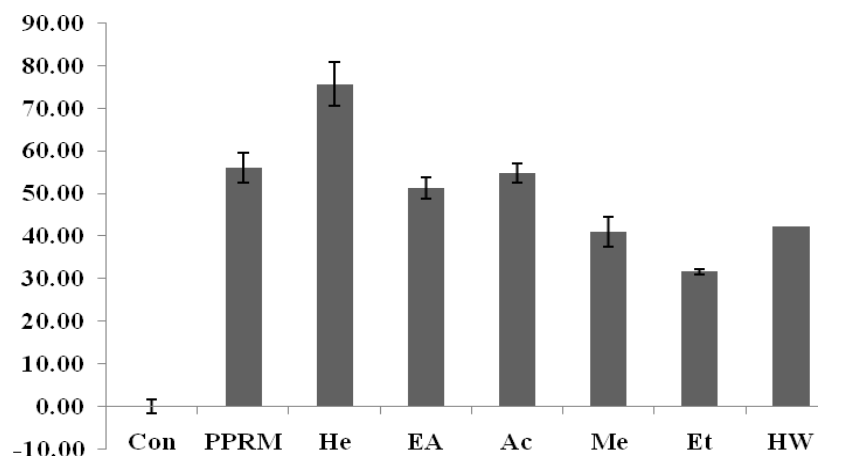

Figure 3. Elastase inhibitory activities in various solvent extracts of C.yabunikkei leaves $(0.5 \mathrm{mg} / \mathrm{mL})$ and Phospharamidon (PPRM, $0.5 \mathrm{mg} / \mathrm{mL}$ ), He; hexane, EA; ethylacetate, Ac; acetone, Me; methanol, Et; ethanol, HW; hot water extract. Values were the mean \pm standard deviation $(n=3)$.

\subsection{Identification of Some Active Constituents}

In the present study, several candidates of extracts of C.yabunikkei were analyzed and it was confirmed that several compounds' were present as common constituents inthe C.yabunikkei leaves. This finding is of significance in the use of the plant for industrial purposes.

None of the studies reported so far state the diverse activities and constituents of extracts of C.yabunikkei leaves. Thus, to the best of our knowledge, our present study is the first to report the optimization of the extraction process of pharmaceutically active indicators from C.yabunikkei leaves and the comparison of the antioxidant, xanthine oxidase inhibitory and elastase activities of various extracts.

GC-MS analyses were performed to identify the active constituents from C.yabunikkei leaf with antioxidant, xanthine oxidase inhibitory and elastase inhibitory activities. Typical GC-MS chromatograms of phytochemical contents and their retention times are shown in Table 2. Eight compounds [i.e., $\alpha$-Linolenic acid (16.1\%), Hexadecanoic acid $(11.48 \%)$, Neophytadiene $(2.05 \%)$, D-(-)-Fructofuranose (2.03\%), $\alpha$-Tocopherol (1.65\%), Phytol (1.36\%), $\beta$-Eudesmol $(0.94 \%)$, Guaiol $(0.86 \%)$, and D-(-)-Fructofuranose $(0.68 \%)]$ were mainly identified by GC-MS analysis (Figure 4$)$. As shown in Table 2, several components associated with antioxidant efficacy and xanthine oxidase inhibition were referenced. $\alpha$-Linolenic acid and $\alpha$-Tocopherol were typical substances with both antioxidant effect and xanthine oxidase inhibition. In Table 2, it is considered that the activity of hexane extract is due to the synergistic effect of various substances since a number of active substances are involved in the process of antioxidant and xanthine oxidase inhibition. 
Table 2. Identified substances from the hexane extracts of C.yabunikkei.

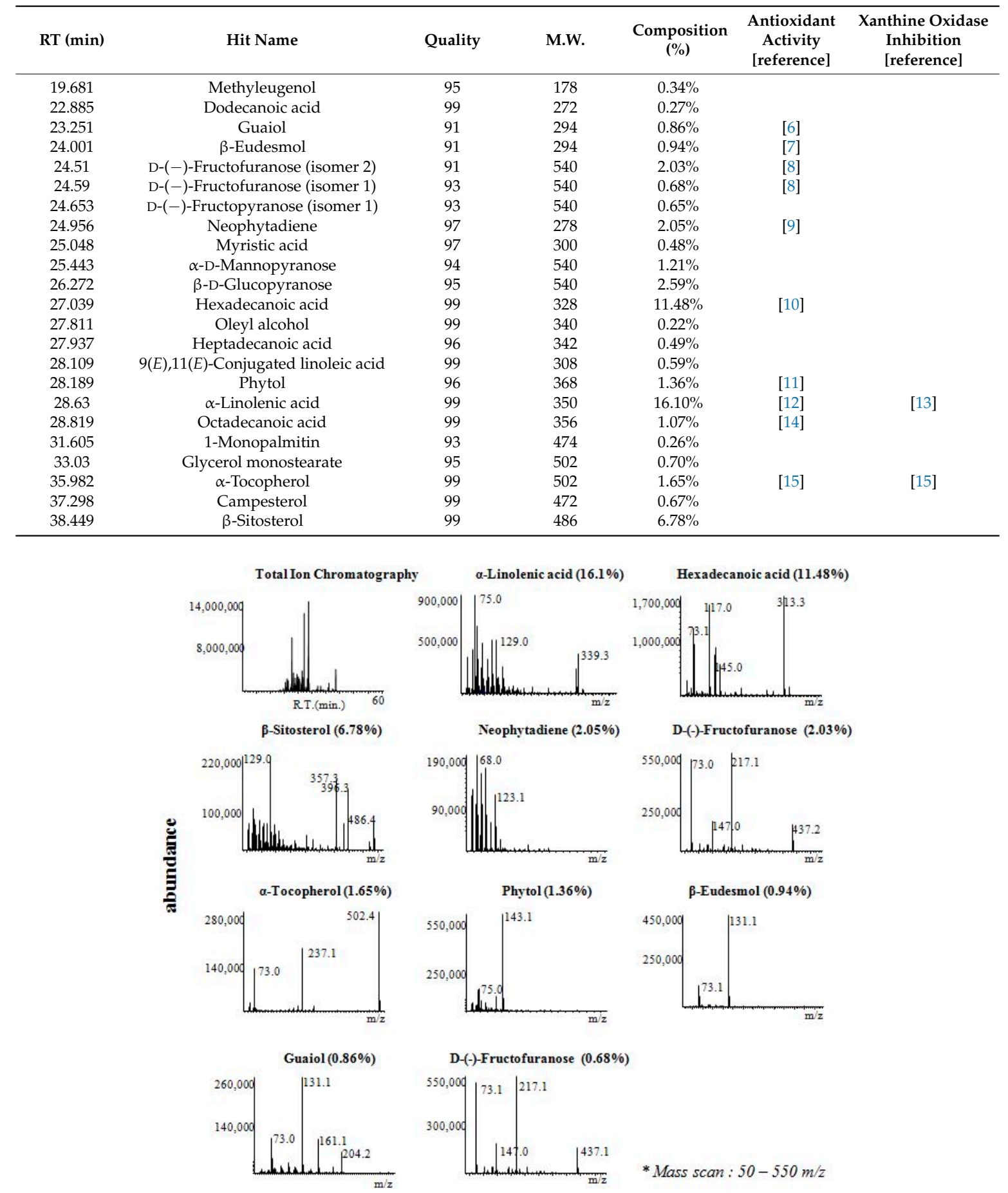

Figure 4. Representative GC-MS chromatogram of extracts of C.yabunikkei leaf.

\section{Experimental Section}

\subsection{Plant Material and Extract Preparation}

C.yabunikkei leaves were supplied by Wando Arboretum (Wando, Korea). A voucher specimen (MNUCSS-CY-01) was deposited at the Mokpo National University (Muan, Korea). Air-dried and powdered C.yabunikkei leaves $(20 \mathrm{~g})$ were subjected to extraction twice with hexane, ethyl acetate, acetone, ethanol, and methanol $(100 \mathrm{~mL})$ at room temperature for $48 \mathrm{~h}$ or subjected to extraction with 
hot water $\left(100{ }^{\circ} \mathrm{C}\right)$ for $4 \mathrm{~h}$. The resultant solution was evaporated, dried, and stored at $-20^{\circ} \mathrm{C}$ for further experiments.

\subsection{DPPH Free Radical Assay}

Antioxidant activity of the sample was determined following a 2,2-diphenyl-1-picrylhydrazyl (DPPH) radical scavenging assay. Briefly, sample solution $(1 \mathrm{~mL})$ containing 1 to $20 \mathrm{mg}$ of sample was added to $0.4 \mathrm{mM}$ DPPH sample solution $(1 \mathrm{~mL})$ and mixed. The mixture was allowed to react at room temperature in the dark for $10 \mathrm{~min}$. Absorbance value at $517 \mathrm{~nm}$ was measured using a microplate reader (Perkin Elmer, Waltham, MA, USA). The DPPH free radical scavenging activities of samples were compared in terms of their $\mathrm{IC}_{50}(\mu \mathrm{g} / \mathrm{mL})$ values [5].

\subsection{Reducing Power}

The reducing power of the sample was determined following a modified reducing power assay method. The sample $(0.1 \mathrm{~mL})$ was added to $0.2 \mathrm{M}$ sodium phosphate buffer $(0.5 \mathrm{~mL})$ and $1 \%$ potassium ferricyanide $(0.5 \mathrm{~mL})$ followed by incubation at $50{ }^{\circ} \mathrm{C}$ for $20 \mathrm{~min}$. Subsequently, $10 \%$ trichloroacetic acid solution $(0.5 \mathrm{~mL})$ was added to the reaction mixture followed by centrifugation at $12,000 \mathrm{rpm}$ for $10 \mathrm{~min}$. The supernatant was mixed with distilled water $(0.5 \mathrm{~mL})$ and $0.1 \%$ iron (III) chloride solution $(0.1 \mathrm{~mL})$. The absorbance value of the resulting solution was measured at $700 \mathrm{~nm}$. Reducing powers of samples were expressed as vitamin C equivalents [5].

\subsection{Determination of Total Phenolic Content}

The total phenolic content was determined using Folin-Ciocalteu assay [5]. Sample (1 mL) containing $5 \mathrm{mg}$ of sample or standard was mixed with $1 \mathrm{~mL}$ of $2 \%$ sodium carbonate solution and $1 \mathrm{~mL}$ of $10 \%$ Folin-Ciocalteu's phenol reagent. After incubating the mixture at room temperature for $10 \mathrm{~min}$, the absorbance was measured at $750 \mathrm{~nm}$ using a microplate reader (Perkin Elmer, Waltham, MA, USA) and compared with the calibration curve of gallic acid. Results were expressed as milligrams of gallic acid equivalents per gram of sample [5].

\subsection{Determination of Xanthine Oxidase Inhibitory Activity}

Xanthine oxidase inhibitory activity was measured by monitoring uric acid formation in the xanthine oxidase system as described previously [5]. The assay system consisted of $0.6 \mathrm{~mL}$ phosphate buffer (100 mM; pH 7.4), $0.1 \mathrm{~mL}$ sample, $0.1 \mathrm{~mL}$ xanthine oxidase $(0.2 \mathrm{U} / \mathrm{mL})$, and $0.2 \mathrm{~mL}$ xanthine ( $1 \mathrm{mM}$; dissolved in $0.1 \mathrm{~N} \mathrm{NaOH}$ ). The reaction was initiated by adding the enzyme with or without inhibitors. A $0.2 \mathrm{~mL}$ aliquot of $1 \mathrm{~N} \mathrm{HCl}$ was used to stop the enzymatic reaction. Allopurinol was used as a positive control. The absorbance of the reaction mixture was measured at $290 \mathrm{~nm}$ using a microplate reader (Perkin Elmer, Waltham, MA, USA).

\subsection{Determination of Elastase Inhibitory Activity}

The assay was modified and performed according to the method of Chiocchio et al. [16]. Briefly, $10 \mu \mathrm{L}$ elastase from porcine pancreas $(10 \mu \mathrm{g} / \mathrm{mL})$ was mixed with 90 of $0.2 \mathrm{M}$ Tris- $\mathrm{HCl}, 100 \mathrm{uL}$ of STANA (2.5 mM, N-Succinyl-Ala-Ala-Ala-p-nitroanilide), and $50 \mu \mathrm{L}$ of sample at $37{ }^{\circ} \mathrm{C}$ for $30 \mathrm{~min}$. After completion of the reaction, the supernatant was centrifuged at 15,000 rpm for $10 \mathrm{~min}$. The absorbance of the reaction mixture was measured at $405 \mathrm{~nm}$ using a microplate reader (Perkin Elmer, Waltham, MA, USA). Phosphoramidon was used as a positive control.

\subsection{Chemical Profiling by GC-MS Analysis}

The molecular mass fragmental scanning of active constituents from C.yabunikkei leaf using GC-MS was performed based on a previously reported method with moderate modifications [14]. Briefly, Agilent 7890 gas chromatography (GC) and Agilent 5975 quadrupole mass spectrometry (MS) 
system (Agilent Technologies, Palo Alto, CA, USA) was utilized to analyze molecular mass fragments (50-550 amu) of C.yabunikkei leaf. The mass fragments were ionized under electron ionization (EI) conditions after an Agilent HP-5MS fused silica capillary column (30 mm $l . \times 0.25 \mathrm{~mm}$ i.d., 0.25- $\mu \mathrm{m}$ film thickness). GC oven was thermally programmed as isothermal at $65^{\circ} \mathrm{C}$ for $10 \mathrm{~min}$ and $10 \mathrm{~min}^{-1}$ to 300 with helium (He) as a carrier gas. All the scanned mass spectra were compared with the data system library (NIST 2017).

\section{Conclusions}

The present study reveals that hot water extract of C.yabunikkei leaf possesses antioxidant activity and hexane extract possesses xanthine oxidase and elastase inhibitory activities. In addition, it is hypothesized that the photochemicals present in the C.yabunikkei leaf might be responsible for the biological activities. The results of this study provide an excellent foundation for the future development of C.yabunikkei leaf-based dietary or medicinal preparations.

Author Contributions: S.Y.-S., M.-S.B. and S.-H.S. performed the experiments and S.-S.C. statistically analyzed the data.

Funding: This research was funded the National Research Foundation of Korea (NRF) grant funded by the Korea government (MSIP; Ministry of Science, ICT \& Future Planning) (No. NRF-2017R1C1B5015187) and Wando county (2017120B312-00).

Conflicts of Interest: The authors declare no conflict of interest.

\section{References}

1. Seo, J.H.; Kim, J.E.; Shim, J.H.; Yoon, G.; Bang, M.A.; Bae, C.S.; Lee, K.J.; Park, D.H.; Cho, S.S. HPLC Analysis, Optimization of Extraction Conditions and Biological Evaluation of Corylopsis coreana Uyeki Flos. Molecules 2016, 21, 94. [CrossRef] [PubMed]

2. Yoon, I.S.; Park, D.H.; Bae, M.S.; Oh, D.S.; Kwon, N.H.; Kim, J.E.; Choi, C.Y.; Cho, S.S. In vitro and in vivo studies on Quercus acuta Thunb. (Fagaceae) extract: Active constituents, serum uric acid suppression, and xanthine oxidase inhibitory activity. Evid.-Based Complement. Altern. Med. 2017, 2017, 4097195. [CrossRef] [PubMed]

3. Yoon, I.S.; Park, D.H.; Kim, J.E.; Yoo, J.C.; Bae, M.S.; Oh, D.S.; Shim, J.H.; Choi, C.Y.; An, K.W.; Kim, E.I.; et al. Identification of the biologically active constituents of Camellia japonica leaf and anti-hyperuricemic effect in vitro and in vivo. Int. J. Mol. Med. 2017, 39, 1613-1620. [CrossRef] [PubMed]

4. Yoon, I.S.; Park, D.H.; Ki, S.H.; Cho, S.S. Effects of extracts from Corylopsis coreana Uyeki (Hamamelidaceae) flos on xanthine oxidase activity and hyperuricemia. J. Pharm. Pharmacol. 2016, 68, 1597-1603. [CrossRef] [PubMed]

5. Song, S.H.; Ki, S.H.; Park, D.H.; Moon, H.S.; Lee, C.D.; Yoon, I.S.; Cho, S.S. Quantitative analysis, extraction optimization, and biological evaluation of Cudrania tricuspidata leaf and fruit extracts. Molecules 2017, 22, 1489. [CrossRef] [PubMed]

6. Pino, J.A.; Regalado, E.L.; Rodriguez, J.L.; Fernandez, M.D. Phytochemical analysis and in vitro free-radical-scavenging activities of the essential oils from leaf and fruit of Melaleuca leucadendra L. Chem. Biodivers. 2010, 7, 2281-2288. [CrossRef] [PubMed]

7. Larayetan, R.A.; Okoh, O.O.; Sadimenko, A.; Okoh, A.I. Terpene constituents of the aerial parts, phenolic content, antibacterial potential, free radical scavenging and antioxidant activity of Callistemon citrinus (Curtis) Skeels (Myrtaceae) from Eastern Cape Province of South Africa. BMC Complement. Altern. Med. 2017, 17, 292. [CrossRef] [PubMed]

8. Zhang, C.R.; Aldosari, S.A.; Vidyasagar, P.S.; Nair, K.M.; Nair, M.G. Antioxidant and anti-inflammatory assays confirm bioactive compounds in Ajwa date fruit. J. Agric. Food Chem. 2013, 61, 5834-5840. [CrossRef] [PubMed]

9. Cheng, M.C.; Chang, W.H.; Chen, C.W.; Li, W.W.; Tseng, C.Y.; Song, T.Y. Antioxidant Properties of Essential Oil Extracted from Pinus morrisonicola Hay Needles by Supercritical Fluid and Identification of Possible Active Compounds by GC/MS. Molecules 2015, 20, 19051-19065. [CrossRef] [PubMed] 
10. Henry, G.E.; Momin, R.A.; Nair, M.G.; Dewitt, D.L. Antioxidant and cyclooxygenase activities of fatty acids found in food. J. Agric. Food Chem. 2002, 50, 2231-2234. [CrossRef] [PubMed]

11. Santos, C.C.; Salvadori, M.S.; Mota, V.G.; Costa, L.M.; de Almeida, A.A.; de Oliveira, G.A.; Costa, J.P.; de Sousa, D.P.; de Freitas, R.M.; de Almeida, R.N. Antinociceptive and Antioxidant Activities of Phytol In Vivo and In Vitro Models. Neurosci. J. 2013, 2013, 949452. [CrossRef] [PubMed]

12. Fagali, N.; Catala, A. Antioxidant activity of conjugated linoleic acid isomers, linoleic acid and its methyl ester determined by photoemission and DPPH techniques. Biophys. Chem. 2008, 137, 56-62. [CrossRef] [PubMed]

13. Songur, A.; Sarsilmaz, M.; Sogut, S.; Ozyurt, B.; Ozyurt, H.; Zararsiz, I.; Turkoglu, A.O. Hypothalamic superoxide dismutase, xanthine oxidase, nitric oxide, and malondialdehyde in rats fed with fish omega-3 fatty acids. Prog. Neuropsychopharmacol. Biol. Psychiatry 2004, 28, 693-698. [CrossRef] [PubMed]

14. Zheng, X.; Wang, H.; Zhang, P.; Gao, L.; Yan, N.; Li, P.; Liu, X.; Du, Y.; Shen, G. Chemical Composition, Antioxidant Activity and alpha-Glucosidase Inhibitory Activity of Chaenomeles Speciosa from Four Production Areas in China. Molecules 2018, 23, 2518. [CrossRef] [PubMed]

15. Mohd Fahami, N.A.; Ibrahim, I.A.; Kamisah, Y.; Ismail, N.M. Palm vitamin E reduces catecholamines, xanthine oxidase activity and gastric lesions in rats exposed to water-immersion restraint stress. BMC Gastroenterol. 2012, 12, 54. [CrossRef] [PubMed]

16. Chiocchio, I.; Mandrone, M.; Sanna, C.; Maxia, A.; Tacchini, M.; Poli, F. Screening of a hundred plant extracts as tyrosinase and elastase inhibitors, two enzymatic targets of cosmetic interest. Ind. Crop. Prod. 2018, 122, 498-505. [CrossRef]

Sample Availability: Samples of the compounds are not available from the authors.

(C) 2018 by the authors. Licensee MDPI, Basel, Switzerland. This article is an open access article distributed under the terms and conditions of the Creative Commons Attribution (CC BY) license (http://creativecommons.org/licenses/by/4.0/). 